\begin{tabular}{|c|l|}
\hline Title & Nonlocal biphoton generation in a Werner state from a single semiconductor quantum dot \\
\hline Author(s) & Kumano, Hidekazu; Nakajima, Hideaki; Kuroda, Takashi; Mano, Takaaki; Sakoda, Kazuaki; Suemune, Ikuo \\
\hline Citation & $\begin{array}{l}\text { Physical review B, 91(20), 205437-1-205437-6 } \\
\text { https://doi.org/10.1103/PhysRevB.91.205437 }\end{array}$ \\
\hline Issue Date & 2015-05-27 \\
\hline Doc URL & http://hdl.handle.net/2115/59521 \\
\hline Rights & @2015A merican Physical Society \\
\hline Type & article \\
\hline File Information & PhysRevB.91.205437.pdf \\
\hline
\end{tabular}

Instructions for use 


\title{
Nonlocal biphoton generation in a Werner state from a single semiconductor quantum dot
}

\author{
H. Kumano, ${ }^{1, *}$ H. Nakajima, ${ }^{1}$ T. Kuroda, ${ }^{2}$ T. Mano, ${ }^{2}$ K. Sakoda, ${ }^{2}$ and I. Suemune ${ }^{1}$ \\ ${ }^{1}$ Research Institute for Electronic Science, Hokkaido University, Sapporo 001-0021, Japan \\ ${ }^{2}$ National Institute for Materials Science, 1 Namiki, Tsukuba 305-0044, Japan \\ (Received 1 August 2014; revised manuscript received 10 May 2015; published 26 May 2015)
}

\begin{abstract}
We demonstrate the generation of a Werner-like state from a single semiconductor quantum dot. The tomographic analysis with temporal gating brings us to a systematic understanding of the relation between the time evolution of quantum correlation and a set of parameters characterizing the exciton states, including fine-structure splitting and cross-dephasing time. The Werner state relates the Bell's parameter in the Clauser, Horne, Shimony, and Holt inequality with a fidelity, which facilitates the evaluation of nonlocality.

DOI: 10.1103/PhysRevB.91.205437

PACS number(s): 78.67.Hc, 03.65.Wj, 03.67.Bg, 78.20.Bh
\end{abstract}

\section{INTRODUCTION}

The quantum biparticle state is the simplest physical system that can exhibit profound quantum-mechanical phenomena such as entanglement between causally independent particles [1] and nonlocality [2,3]. These properties are at the heart of quantum information and communication technology, providing unconditional security [4,5]. For two-qubit pure states, entanglement and nonlocality are equivalent [6]. In practice, however, all the systems are inevitably driven into mixed states because any system is more or less open to its environment and subject to loss and decoherence. Although the relation between entanglement, nonlocality, and teleportation fidelity is not fully understood for general two-qubit mixed states $[7,8]$, one of the mixed entangled states, the so-called Werner state $[9,10]$, has been widely investigated because of its widespread ramifications. For example, there exist bipartite mixed states that are entangled but do not violate any Bell-type inequalities [9], and the Werner states can be regarded as maximally entangled mixed states of two-qubit systems whose degree of entanglement cannot be increased by any unitary operations [11]. Moreover, all the entangled Werner states are useful for teleportation [12]. Therefore, the generation of Werner states is of significant importance for practical biphoton sources employed in the field of quantum information and communication technology.

For quantum photon sources with parametric downconversion [13,14], signal and idler photons have an intrinsic quantum-mechanical correlation. Biphoton states have been extensively studied with quantum state tomography [15-17], and the formation of the Werner state was proved by introducing polarization diffusers [18,19]. On the other hand, for quantum-dot (QD) photon sources [20], despite their potential feasibility for quasideterministic operations, biphoton generation with quantum correlation is not straightforward. Lowering the symmetry of the exciton confinement potential results in an anisotropic $e-h$ exchange and brings fine-structure splitting (FSS) in the bright exciton states [21]. The resultant whichpath information and the time-integrated measurement hinder quantum correlation by breaking the superposition between the two decaying paths $H_{X X} H_{X}$ and $V_{X X} V_{X}$ for the neutral biexciton $\left(X X^{0}\right)$-exciton $\left(X^{0}\right)$ cascading process [22]. To date,

*kumano@es.hokudai.ac.jp to suppress the which-path information for a selected QD, electric [23-25], magnetic [26], and strain [27,28] fields, as well as spectral [29] or temporal [30,31] filtering, were applied; thence, polarization-entangled [23-30] photon-pair generation has been achieved. Moreover, the strengthening nonlocality of the photon pair has also been demonstrated with temporal filtering [31]. However, biphoton states generated from QDbased sources are argued basically from the viewpoint of a state being entangled (or nonlocal) or not, and further details about the biphoton states against all the physically possible biphoton mixed states still remain elusive.

In this paper, biphoton states via $X X^{0}-X^{0}$ cascading emission from a QD are systematically examined on the basis of an analytical density matrix for the excitonic system given by Hudson et al. [32]. As a result, we have established a quantummechanical description of the biphoton state obtained from a quantum-dot emitter as a Werner state. The Werner state distinguishes the obtained biphoton state from the generally allowed biphoton mixed states and relates the so-called Bell's $S$ parameter to a widely used fidelity. The relation between the biphoton states and fundamental parameters to determine the underlying dynamics in the exciton states is also clarified by comparing an analytically evaluated density matrix with a tomographically reconstructed matrix by applying temporal gating.

\section{DOT EMITTER PREPARATION AND BIPHOTON-STATE ANALYSIS}

As a biphoton emitter, we employed an unstrained GaAs QD formed on a lattice-matched $\mathrm{Al}_{0.3} \mathrm{Ga}_{0.7} \mathrm{As}$ barrier layer grown on a GaAs (111)A substrate by droplet epitaxy [33,34]. Based on $C_{3 v}$ symmetry of the (111) surface with identical in-plane covalent bonds, highly symmetric "as-grown” QDs emitting biphoton states with quantum correlation are demonstrated [35-38]. Further details on the growth condition and the sample structure are given elsewhere [33,35]. For optical characterization, the sample was cooled to $9 \mathrm{~K}$ and a $640 \mathrm{~nm}$ pulsed semiconductor laser was used to pump the $\mathrm{Al}_{0.3} \mathrm{Ga}_{0.7} \mathrm{As}$ barrier continuum.

Figure 1 shows the photoluminescence (PL) spectrum of an isolated GaAs dot. In our samples, QDs typically have four emission lines, i.e., negatively charged excitons $\left(X^{-}\right)$, neutral biexcitons $\left(X X^{0}\right)$, positively charged excitons $\left(X^{+}\right)$, and neutral excitons $\left(X^{0}\right)$ in order of increasing energy. Prior to the biphoton-state analysis using photon correlation 


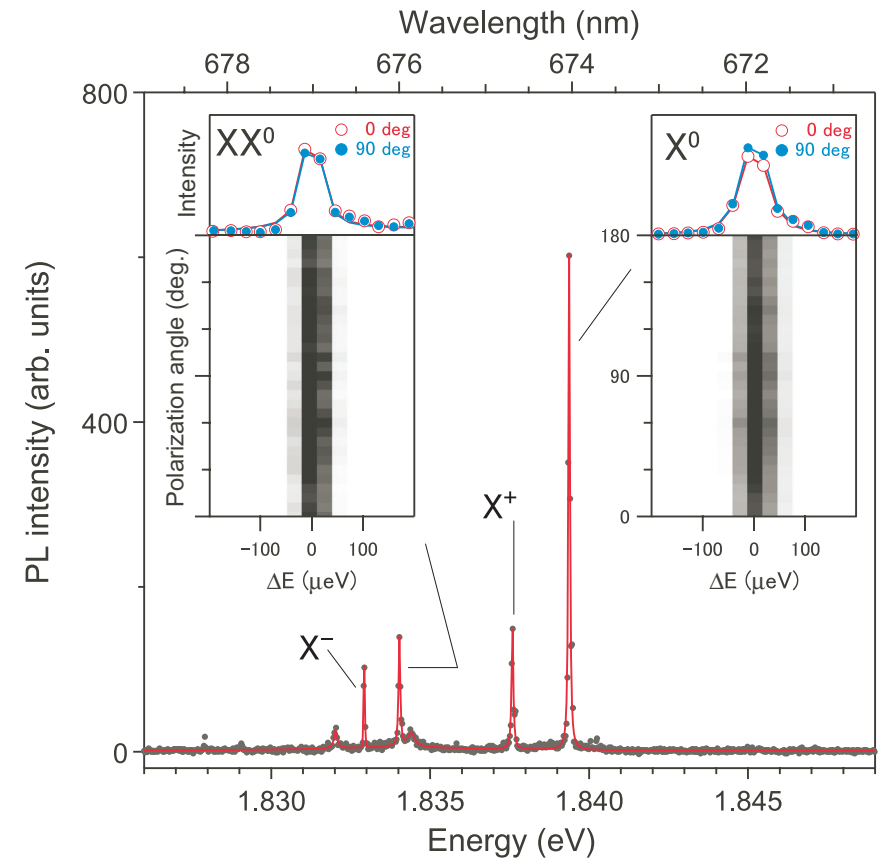

FIG. 1. (Color online) PL spectrum of the isolated GaAs QD at $9 \mathrm{~K}$. Lorentzian fitting is also shown as a red curve. The biphoton state is generated from the $\left|X X^{0}\right\rangle \rightarrow\left|X^{0}\right\rangle \rightarrow \mid$ Vac $\rangle$ cascading process. Inset: Detection polarization dependence (in linear basis) of the emission energy for the $X^{0}$ and $X X^{0}$ lines.

measurements, we have analyzed the PL spectra as a function of polarization angle, as shown in the insets of Fig. 1, which reveals that the FSS value is smaller than the linewidth. In addition, the amplitude of the peak energy difference in the $X X^{0}$ and $X^{0}$ lines extracted with a sinusoidal fitting [31] was below the measurement uncertainly of $0.40 \mu \mathrm{eV}$. Biphoton correlation was investigated with a pair of cascadingly emitted $X X^{0}$ and $X^{0}$ photons by analyzing the coincidence counts using a time-to-digital converter (TDC). In this study, we have measured coincidence with $6^{2}$ polarization configurations, i.e., $\{H, V, D, A, R, L\}$ polarizations for each line, where $H, V, D$, $A, R$, and $L$ indicate projections along the linear laboratory horizontal, vertical, diagonal $\left(+45^{\circ}\right.$ from $\left.H\right)$, antidiagonal $\left(-45^{\circ}\right.$ from $\left.H\right)$, right-handed circular, and left-handed circular polarizations, respectively. Figure 2 shows a typical line shape of the coincidence counts, and all the unprocessed data measured with the 36 configurations are shown in the Appendix (Fig. 6).

To analyze the biphoton state from the QD, density matrices were tomographically reconstructed from the 36 datasets entailing the maximum likelihood method [15]. The degree of mixedness and entanglement are evaluated in terms of linear entropy $\left(S_{L}\right)$ [39] and tangle $(T)$ [40], respectively. These measurements can be calculated explicitly from the obtained density matrix $\rho$ as $S_{L}=\frac{4}{3}\left(1-\operatorname{Tr} \rho^{2}\right)$, and $T=\left[\max \left(\lambda_{1}-\lambda_{2}-\lambda_{3}-\lambda_{4}, 0\right)\right]^{2}$, where $\lambda_{i}(i=1,2$, $3,4)$ is the square root of the eigenvalues in decreasing order of magnitude of the spin-flipped density matrix operator $R=\rho\left(\sigma^{y} \otimes \sigma^{y}\right) \rho^{*}\left(\sigma^{y} \otimes \sigma^{y}\right)$, where $\sigma^{y}$ is one of Pauli's operators, and the asterisk indicates complex conjugation. Biphoton states can be displayed in the $S_{L}-T$ plane, as shown

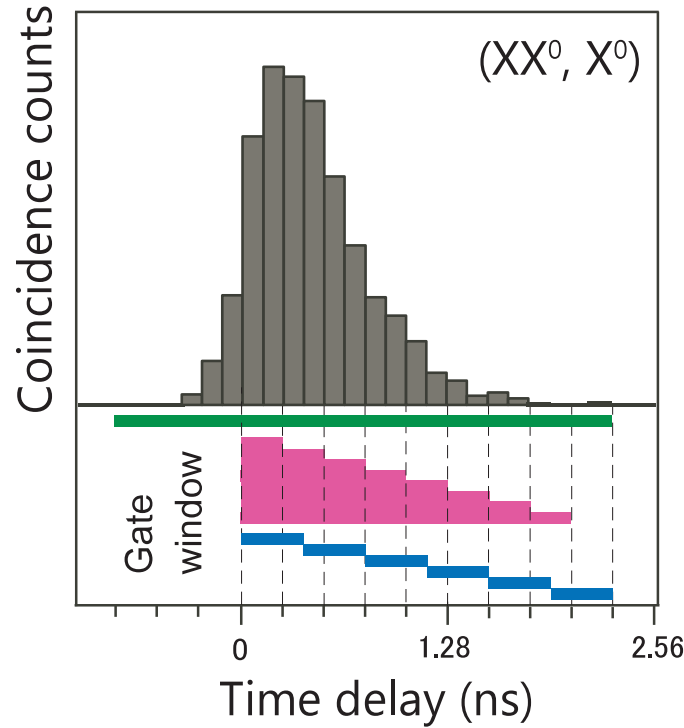

FIG. 2. (Color online) Upper panel: Typical line shape of our histogram of the coincidence counts for the $X X^{0}-X^{0}$ cascade. Lower panel: Integrating ranges for obtaining coincidence counts. The green stripe covers the entire peak with an integration time of $3.072 \mathrm{~ns}$, and we refer to it as without gating. Two types of temporal gating, (i) widening gate (red) and (ii) shifting gate (blue), are employed to evaluate the time evolution of the biphoton states generated from a highly symmetric QD. $\Delta t_{g}$ is 256 and 384 ps, respectively. The time origin was determined to the point that provides the highest fidelity to $\left|\Phi^{+}\right\rangle$. Biphoton density matrices are tomographically reconstructed with 36 datasets for each temporal gating.

in Fig. 3(a), wherein all the physically allowed biphoton states will be mapped on the white region below the dashed line. In this plane, $\left(S_{L}, T\right)=(0,1)$ represents the maximally entangled states, while $\left(S_{L}, T\right)=(1,0)$ represents totally mixed states. First, we analyzed the biphoton state without temporal gating. In this case, outputs from the TDC were integrated over the range covering the entire peak (the green stripe in the lower panel of Fig. 2), and the resultant biphoton state is plotted as a green square in Fig. 3(a). The state is on the Werner curve indicated by the solid line. This is a clear manifestation that the biphoton state emitted from the QD belongs to the Werner state. Another important consequence of this analysis is that, assuming the biphoton state is the Werner state, one can readily see that the state is entangled for $S_{L}<8 / 9$ and further violates the local hidden variable model for $S_{L}<1 / 2$ [17]. In the present case, we have $S_{L}=0.436(<1 / 2)$ and $T=0.382$. Thus, we can conclude that the generated biphoton state from the present QD is nonlocal, which is consistent with a direct demonstration of violating the Clauser, Horne, Shimony, and Holt (CHSH) version of Bell's inequality [3] without any fields or filtering [35].

\section{TIME EVOLUTION OF DENSITY MATRIX}

The present highly symmetric QD emitter provides biphoton states endowed with a high degree of entanglement and nonlocality even without temporal gating. This achievement sheds light on the dynamics underlying the intermediate exciton states, such as coherent evolution of the state vector and 

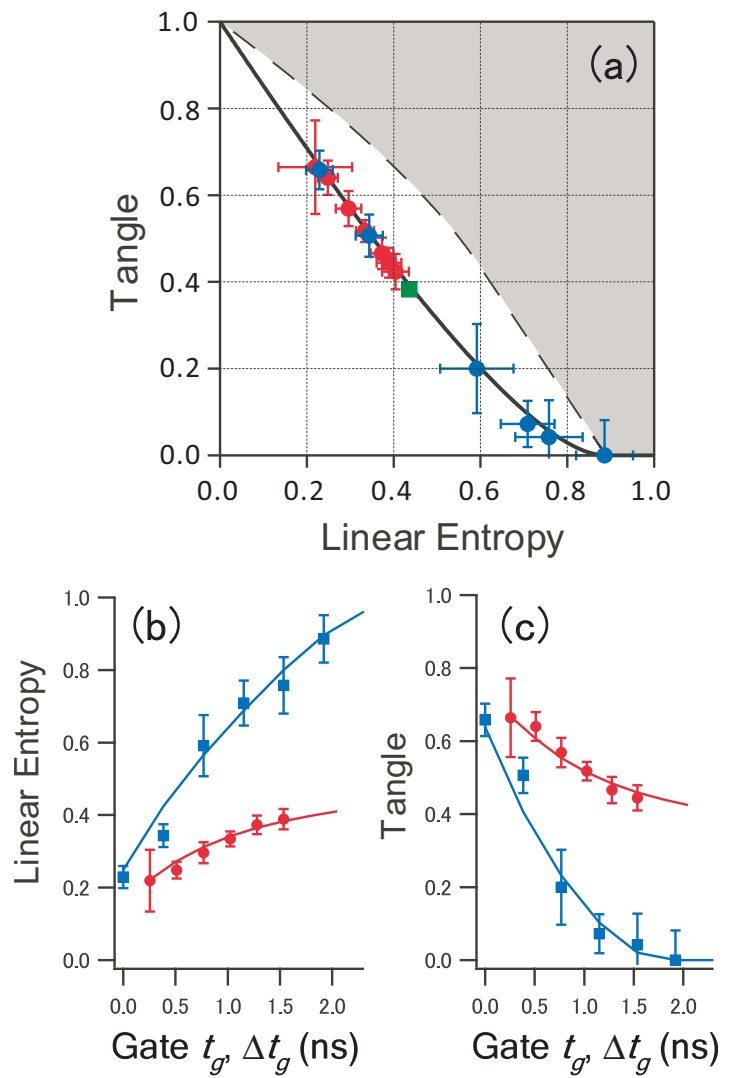

FIG. 3. (Color online) Time evolution of the biphoton mixed state from a QD mapped on the $S_{L}-T$ plane. The Werner state is denoted by a solid line. (a) Experimentally obtained states via quantum tomography with 36 polarization bases are shown by red (blue) circles employing the widening (shifting) gate. A green square exhibits the biphoton state without temporal gating. The corresponding time evolution of the biphoton state in terms of (b) linear entropy and (c) tangle is shown by solid lines. The horizontal axis in (b) and (c) is $\Delta t_{g}\left(t_{g}\right)$ for the widening (shifting) gate.

the relaxation processes involved. With a finite delay between a pair of $X X^{0}-X^{0}$ photon generations and the FSS (denoted by $S$ ), the possible biphoton pure state from the emitter is expressed as a Bell state, $\left|\Phi^{+}\right\rangle=\frac{1}{\sqrt{2}}(|H H\rangle+|V V\rangle)$, with a relative phase of $\exp (i S t / \hbar)$ gained in the dwell time $t$ in the intermediate exciton state. The probability of generating the state from the QD emitter with an exciton lifetime of $\tau_{r}$, at a time between $t$ and $t+d t$, is given by $\frac{1}{\tau_{r}} \exp \left(-t / \tau_{r}\right) d t$ [32]. Therefore, the biphoton state generated in a time duration of $\left[t_{g}, t_{g}+\Delta t_{g}\right]$ is

$$
\hat{\rho}=\int_{t_{g}}^{t_{g}+\Delta t_{g}} \frac{1}{\tau_{r}} \exp \left(-t / \tau_{r}\right) \hat{\rho}_{\text {pure }}(t) d t,
$$

where $\hat{\rho}_{\text {pure }}(t)$ is the density matrix for the pure biphoton state generated at time $t$. This is the fruitful extension from a steady-state density matrix [32], with which we can explore the time evolution of the generated biphoton states in conjunction with exciton dynamics. To construct the general biphoton density matrix, we first consider the effects of spin scattering and background (uncorrelated) light by taking a convex combination of the $\hat{\rho}$ and totally mixed (uncorrelated) state of $\frac{1}{4} I \otimes I$, where $I$ indicates the identity operator of a single qubit. The ratio of spin scattering (characteristic time $\tau_{s s}$ ) to radiative recombination and the fraction of photon pairs stemming from the QD $k$ define the weight of $\hat{\rho}$ as $p \equiv k /\left(1+\tau_{r} / \tau_{s s}\right)$. The parameter $k$ specifying the background contribution will be redefined later. To describe the general biphoton mixed state from the QD including relaxation processes, $1 / \tau_{r}$ in the exponential function in Eq. (1) should be extended properly, so that the population with copolarized components $(|H H\rangle$ and $|V V\rangle)$ decay with a rate of $1 / \tau_{r}+1 / \tau_{s s}$, and decoherence takes place through the further introduced parameter $\tau_{H V}$ in the off-diagonal elements of the matrix to characterize the cross dephasing $[32,41]$. Thus, we obtain the density matrix for the biphoton mixed state with the rectilinear bases of $|H H\rangle,|H V\rangle,|V H\rangle,|V V\rangle$ as

$$
\underline{\rho}=\frac{1}{4}\left(\begin{array}{cccc}
1+p & 0 & 0 & 2 p I_{c}^{*} / I_{0} \\
0 & 1-p & 0 & 0 \\
0 & 0 & 1-p & 0 \\
2 p I_{c} / I_{0} & 0 & 0 & 1+p
\end{array}\right),
$$

where

$$
\begin{gathered}
I_{0}=\int_{t_{g}}^{t_{g}+\Delta t_{g}} \frac{1}{\tau_{r}} e^{-t\left(1 / \tau_{r}+1 / \tau_{s s}\right)} d t, \\
I_{c}=\int_{t_{g}}^{t_{g}+\Delta t_{g}} \frac{1}{\tau_{r}} e^{-t\left(1 / \tau_{r}+1 / \tau_{s s}+1 / \tau_{H V}\right)} e^{i S t / \hbar} d t .
\end{gathered}
$$

If $\left|I_{c} / I_{0}\right|=1$, hence $S=0$ and $p^{\prime} \equiv k /\left(1+\tau_{r} / \tau_{s s}+\right.$ $\left.\tau_{r} / \tau_{H V}\right)=p$ (or equivalently $\tau_{r} / \tau_{H V}=0$ ), the biphoton state $\rho$ reduces to the Werner state and is mapped on a solid line in the $S_{L}-T$ plane in Fig. 3(a) for $0 \leqslant p^{\prime} \leqslant p \leqslant 1$. To analyze the time evolution of the biphoton states from the QD, two kinds of temporal gating, i.e., (i) a widening gate with a constant time increment and (ii) a shifting gate with a fixed width, were employed in a complementary manner. Temporal gatings used for reconstructing the biphoton density matrices are illustrated in the lower panel of Fig. 2. To probe the coherent evolution, since the phase rotates as the monitoring time increases, the widening gate can be more sensitive. On the other hand, a shifting gate is preferable to evaluate the relaxation dynamics. An example of the time evolution of the density matrix is illustrated in Fig. 4.

In Fig. 3(a), the experimentally reconstructed biphoton states with the temporal gating are summarized as red (blue) circles for the widening (shifting) gate. The error bars in each point were estimated by taking into account the error propagation in analytically calculating $S_{L}$ and $T$ [42]. By narrowing the gate width, the biphoton state moves toward the maximally entangled state $\left(S_{L}=0\right)$ along the Werner curve. With the narrowest gate of $256 \mathrm{ps}$ width, we have $S_{L}=0.219$ and $T=0.664$. For the shifting gate, the biphoton state evolved with time along the Werner curve to a totally mixed state $\left(S_{L}=1\right)$ with an increase in the weight of the mixed state component. This finding indicates that cross dephasing is comparatively slower than the radiative lifetime $\left(p^{\prime} / p \simeq 1\right)$ and that the phase rotation $e^{i S t / \hbar}$ contributes rather weakly to Eq. (4) $(S \simeq 0)$, which suggests that $S \ll \hbar / \Delta t_{g}=1.7 \mu \mathrm{eV}$. The time evolution of the biphoton state is also displayed in a more explicit way for both temporal gatings in terms of $S_{L}$ and $T$ in Figs. 3(b) and 3(c), respectively. The shifting gate shows 

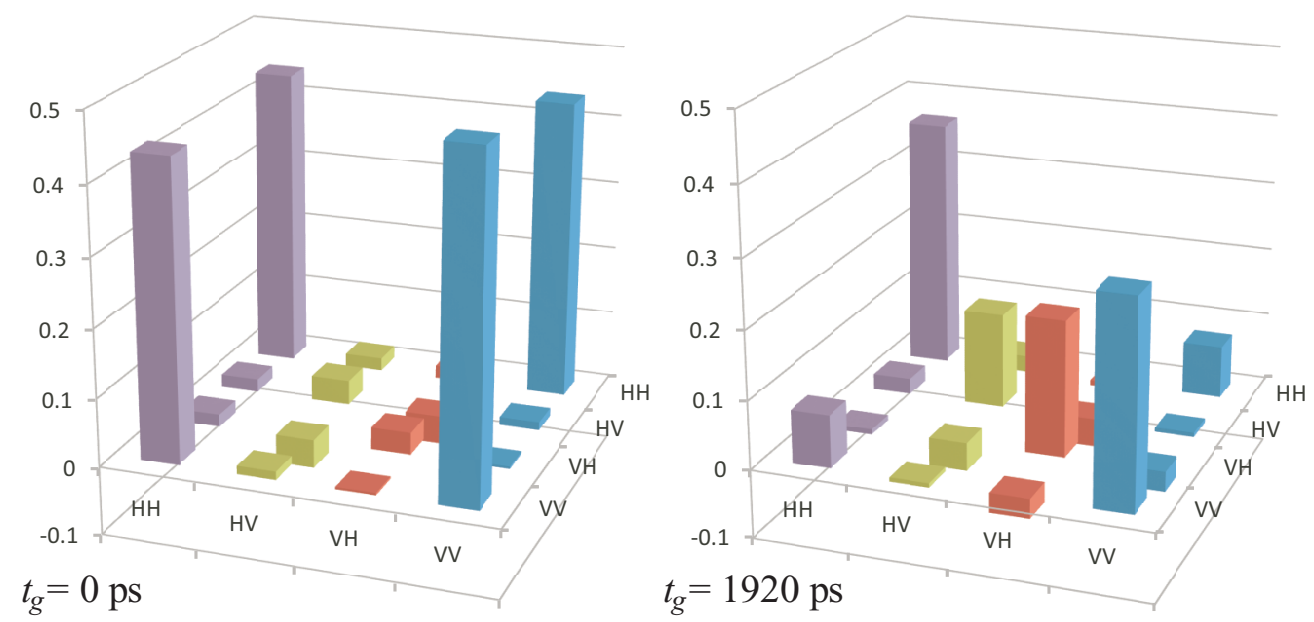

FIG. 4. (Color online) Density matrices (real part) representing two biphoton states obtained at $t_{g}=0$ and 1920 ps for the shifting gate.

stronger degradation with time because of real-time probing of the mixedness and dephasing, and thus the biphoton state is updated without considering the history. To calculate the density matrix, a fraction of photon pairs exclusively from the QD $k$ in $\rho$ is required. For the analysis using temporal gating, since $\overline{\bar{t}}$ e $k$ depends on the adopted gating condition, we introduce an alternative time-independent parameter $d$ to specify the ratio of uncorrelated (background) count rate to the single rate for the QD emission at zero time delay, which is related to $k$ as $k=\left[\frac{S\left(t_{1}, t_{2}\right)}{S\left(t_{1}, t_{2}\right)+d\left(t_{2}-t_{1}\right)}\right]^{2}$, where $S\left(t_{1}, t_{2}\right)=$ $\int_{t_{1}}^{t_{2}} \exp \left(-t / \tau_{r}\right) d t$.

By comparing the experimentally obtained biphoton states using independently measured parameters of $\tau_{r}=560 \mathrm{ps}$ and $\tau_{s s}=2.8 \mathrm{~ns}$ for the identical dot, we have found that $\left(S, \tau_{H V}, d\right)=(0.36 \pm 0.06 \mu \mathrm{eV}, 2.3 \pm 0.5 \mathrm{~ns}, 0.008 \pm 0.004)$ presents the best agreement with the experimental observation in Figs. 3(a)-3(c). Basically, these parameters were obtained to reproduce the upper and lower bounds for the biphoton state with the shifting and widening gates in Fig. 3(a), and the overall behavior in Figs. 3(b) and 3(c). Since the density matrix has full information on the biphoton state, we can deduce the fundamental parameters to characterize the exciton dynamics by analyzing the matrix as a function of the delay time between $X X^{0}$ and $X^{0}$ photogeneration.

\section{TIME EVOLUTION OF FIDELITY}

In Fig. 5, the fidelity $f$ of the experimentally obtained biphoton states to the maximally entangled state $\left|\Phi^{+}\right\rangle$is examined. The fidelity is calculated using $f=\left(1+C_{H / V}+\right.$ $\left.C_{D / A}+C_{R / L}\right) / 4$ for the two temporal gating conditions, where $C_{H / V}, C_{D / A}$, and $C_{R / L}$ are correlation functions in the rectilinear, diagonal, and circular basis, respectively. The overall behavior agrees well with $\left\langle\Phi^{+}|\underline{\underline{\rho}}| \Phi^{+}\right\rangle=[1+p+$ $\left.2 p^{\prime} \operatorname{Re}\left(I_{c} / I_{0}\right)\right] / 4$ using the identical parameters in Figs. 3(b) and 3(c) (solid lines), which indicates that the analytical method presented in this paper is quite useful to attain a comprehensive understanding of the biphoton state from the QD.

The Werner state relates the Bell's $S$ parameter in the CHSH inequality with $f$ [43], which assures that the biphoton states in the Werner state are nonlocal if $f>0.78$. Instead of the direct verification of the violation of Bell's inequality in earlier works [31,35], biphoton mixed states in the Werner state offer a simple criterion to evaluate the nonlocality. Note that even for a zero gate width limit, the fidelity is below unity. This is due to residual nonzero mixedness given by $S_{L} \simeq\left(-2 p^{4} / p^{\prime 2}-p^{2}+\right.$ 3)/3. To realize ideal biphoton pure sources with $S_{L} \simeq 0$, we need $\tau_{r} / \tau_{s s} \simeq 0, \tau_{r} / \tau_{H V} \simeq 0$, and $k \simeq 1$. For this, shortening of $\tau_{r}$ by introducing the Purcel effect [44] with backgroundfree QDs and stabilizing spin states in the intermediate $\left|X^{0}\right\rangle$ state will be crucial.

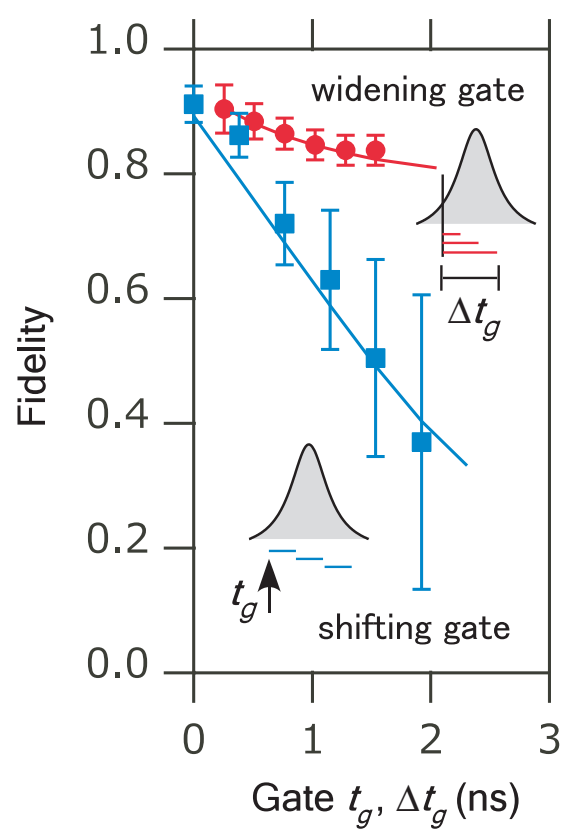

FIG. 5. (Color online) Fidelity $f \quad$ to $\quad$ the $\left|\Phi^{+}\right\rangle=\frac{1}{\sqrt{2}}$ $(|H H\rangle+|V V\rangle)$ for the experimentally obtained biphoton states with the widening (red circles) and shifting (blue squares) gates. Error bars represent one standard deviation. Analytically calculated fidelity employing identical parameters as in Fig. 3 is also displayed as solid lines for each gating condition. $f \simeq(1+p) / 4+p^{2} / 2 p^{\prime}$ for zero gate limit. 


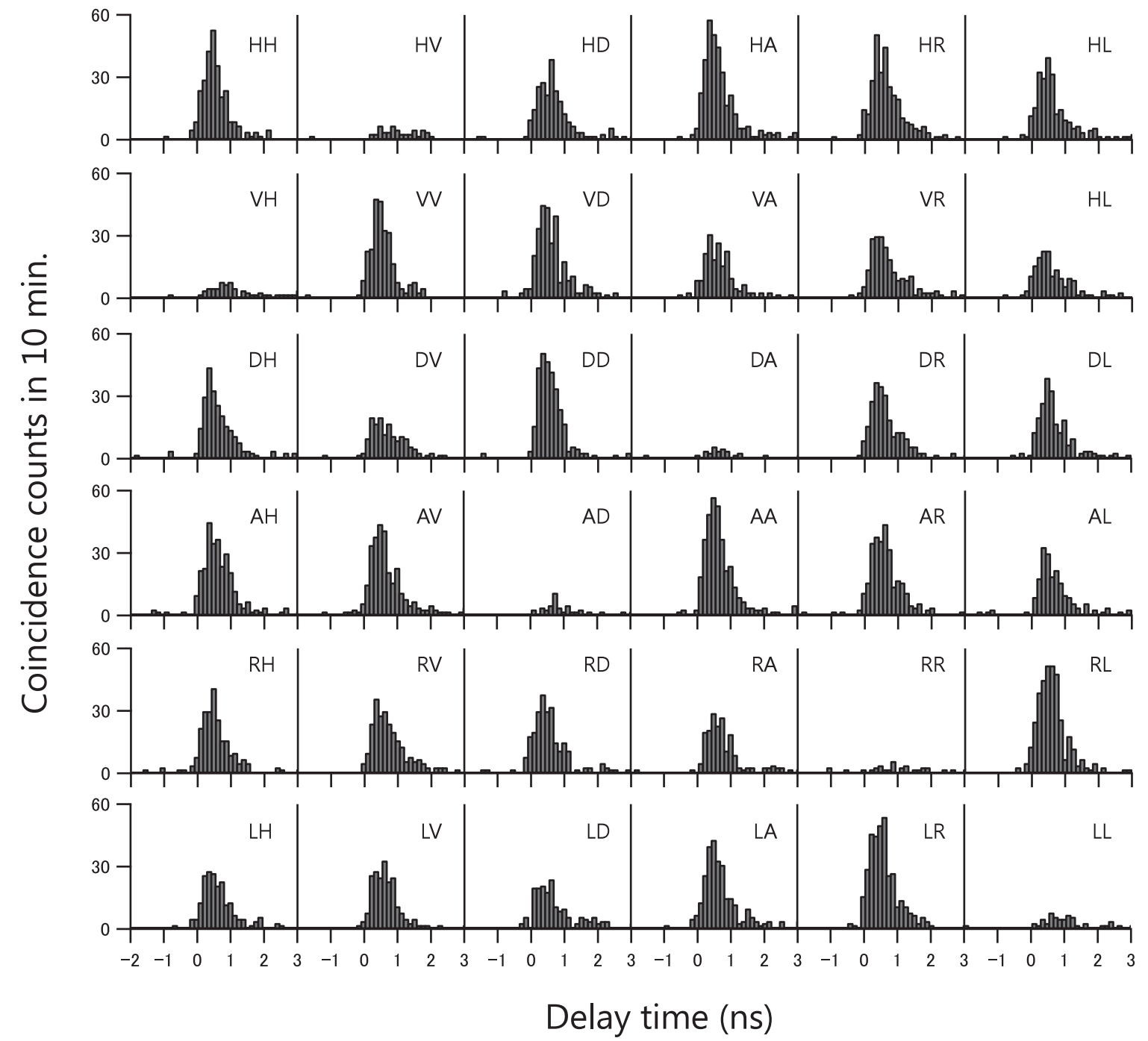

FIG. 6. Photon correlation functions around zero time delay for the 36 polarization configurations used to evaluate the time evolution of the biphoton state. Coincidence counts were accumulated for $10 \mathrm{~min}$.

\section{CONCLUSIONS}

In conclusion, we have demonstrated that the biphoton state generated from a QD via a biexciton-exciton cascade can be well described as a Werner state. The Werner state shows a continuous variation from a nonlocal to local (but entangled) state, and finally to the classical one, which indicates the decreasing weight of the Bell state in the Werner state with a $X X^{0}-X^{0}$ delay time. While the violation of the $\mathrm{CHSH}$ inequality should be experimentally verified to show the nonlocality for a general biphoton mixed state, one can readily conclude, for the Werner state, that it is nonlocal if the fidelity is larger than 0.78 , just as we ensure the entanglement when the fidelity exceeds 0.5 .

\section{ACKNOWLEDGMENTS}

This study was supported in part by the JSPS KAKENHI Grants No. 24310084 and No. 25390011, and the Murata Science Foundation.

\section{APPENDIX: UNPROCESSED COINCIDENCE COUNTS}

In this Appendix, we present additional information on the unprocessed coincidence counts for 36 polarization configurations used in the analysis of the biphoton states emitted from the GaAs QD (Fig. 6 ).
[1] A. Einstein, B. Podolsky, and N. Rosen, Phys. Rev. 47, 777 (1935).

[2] J. S. Bell, Physics 1, 195 (1964).

[3] J. F. Clauser, M. A. Horne, A. Shimony, and R. A. Holt, Phys. Rev. Lett. 23, 880 (1969).
[4] R. Horodecki, P. Horodecki, M. Horodecki, and K. Horodecki, Rev. Mod. Phys. 81, 865 (2009).

[5] N. Gisin, S. Pironio, and N. Sangouard, Phys. Rev. Lett. 105, 070501 (2010).

[6] N. Gisin, Phys. Lett. A 154, 201 (1991). 
[7] F. Buscemi, Phys. Rev. Lett. 108, 200401 (2012).

[8] D. Cavalcanti, A. Acín, N. Brunner, and T. Vértesi, Phys. Rev. A 87, 042104 (2013).

[9] R. F. Werner, Phys. Rev. A 40, 4277 (1989).

[10] Originally, the convex combination of $\left|\Psi^{-}\right\rangle=\frac{1}{\sqrt{2}}$ $(|H V\rangle-|V H\rangle)$ and $I \otimes I$ was called the Werner state, which is invariant under an arbitrary unitary operation for both qubits $U \otimes U$. However, the four Bell states $\left|\Psi^{ \pm}\right\rangle$and $\left|\Phi^{ \pm}\right\rangle$are interchangeable with local operations and the resultant states exhibit the same entanglement properties. Thus the $\left|\Phi^{+}\right\rangle$mixed with $I \otimes I$ is also referred to as the Werner state.

[11] S. Ishizaka and T. Hiroshima, Phys. Rev. A 62, 022310 (2000).

[12] L. Mišta, R. Filip, and J. Fiurášek, Phys. Rev. A 65, 062315 (2002).

[13] P. G. Kwiat, K. Mattle, H. Weinfurter, A. Zeilinger, A. V. Sergienko, and Y. Shih, Phys. Rev. Lett. 75, 4337 (1995).

[14] P. G. Kwiat, E. Waks, A. G. White, I. Appelbaum, and P. H. Eberhard, Phys. Rev. A 60, R773 (1999).

[15] A. G. White, D. F. V. James, W. J. Munro, and P. G. Kwiat, Phys. Rev. A 65, 012301 (2001).

[16] R. Rangarajan, M. Goggin, and P. Kwiat, Opt. Express 17, 18920 (2009).

[17] H. Kumano, K. Matsuda, S. Ekuni, H. Sasakura, and I. Suemune, Opt. Express 19, 14249 (2011).

[18] G. Puentes, A. Aiello, D. Voigt, and J. P. Woerdman, Phys. Rev. A 75, 032319 (2007).

[19] Y.-S. Zhang, Y.-F. Huang, C.-F. Li, and G.-C. Guo, Phys. Rev. A 66, 062315 (2002).

[20] Single Semiconductor Quantum Dots, edited by P. Michler (Springer, Berlin, 2009).

[21] M. Bayer, G. Ortner, O. Stern, A. Kuther, A. A. Gorbunov, A. Forchel, P. Hawrylak, S. Fafard, K. Hinzer, T. L. Reinecke, S. N. Walck, J. P. Reithmaier, F. Klopf, and F. Schäfer, Phys. Rev. B 65, 195315 (2002).

[22] H. Kumano, S. Kimura, M. Endo, H. Sasakura, S. Adachi, S. Muto, and I. Suemune, J. Nanoelectron. Optoelectron. 1, 39 (2006).

[23] M. M. Vogel, S. M. Ulrich, R. Hafenbrak, P. Michler, L. Wang, A. Rastelli, and O. G. Schmidt, Appl. Phys. Lett. 91, 051904 (2007).

[24] A. J. Bennett, M. A. Pooley, R. M. Stevenson, M. B. Ward, R. B. Patel, A. B. de la Giroday, N. Skold, I. Farrer, C. A. Nicoll, D. A. Ritchie, and A. J. Shields, Nat. Phys. 6, 947 (2010).

[25] M. Ghali, K. Ohtani, Y. Ohno, and H. Ohno, Nat. Commun. 3, 661 (2012)
[26] R. M. Stevenson, R. J. Young, P. See, D. G. Gevaux, K. Cooper, P. Atkinson, I. Farrer, D. A. Ritchie, and A. J. Shields, Phys. Rev. B 73, 033306 (2006).

[27] S. Seidl, M. Kroner, A. Högele, K. Karrai, R. J. Warburton, A. Badolato, and P. M. Petroff, Appl. Phys. Lett. 88, 203113 (2006).

[28] R. Trotta, E. Zallo, C. Ortix, P. Atkinson, J. D. Plumhof, J. van den Brink, A. Rastelli, and O. G. Schmidt, Phys. Rev. Lett. 109, 147401 (2012).

[29] N. Akopian, N. H. Lindner, E. Poem, Y. Berlatzky, J. Avron, D. Gershoni, B. D. Gerardot, and P. M. Petroff, Phys. Rev. Lett. 96, 130501 (2006).

[30] R. M. Stevenson, A. J. Hudson, A. J. Bennett, R. J. Young, C. A. Nicoll, D. A. Ritchie, and A. J. Shields, Phys. Rev. Lett. 101, 170501 (2008).

[31] R. J. Young, R. M. Stevenson, A. J. Hudson, C. A. Nicoll, D. A. Ritchie, and A. J. Shields, Phys. Rev. Lett. 102, 030406 (2009).

[32] A. J. Hudson, R. M. Stevenson, A. J. Bennett, R. J. Young, C. A. Nicoll, P. Atkinson, K. Cooper, D. A. Ritchie, and A. J. Shields, Phys. Rev. Lett. 99, 266802 (2007).

[33] T. Mano, M. Abbarchi, T. Kuroda, B. McSkimming, A. Ohtake, K. Mitsuishi, and K. Sakoda, Appl. Phys. Express 3, 065203 (2010).

[34] G. Sallen, B. Urbaszek, M. M. Glazov, E. L. Ivchenko, T. Kuroda, T. Mano, S. Kunz, M. Abbarchi, K. Sakoda, D. Lagarde, A. Balocchi, X. Marie, and T. Amand, Phys. Rev. Lett. 107, 166604 (2011).

[35] T. Kuroda, T. Mano, N. Ha, H. Nakajima, H. Kumano, B. Urbaszek, M. Jo, M. Abbarchi, Y. Sakuma, K. Sakoda, I. Suemune, X. Marie, and T. Amand, Phys. Rev. B 88, 041306 (2013).

[36] R. Singh and G. Bester, Phys. Rev. Lett. 103, 063601 (2009).

[37] A. Schliwa, M. Winkelnkemper, A. Lochmann, E. Stock, and D. Bimberg, Phys. Rev. B 80, 161307 (2009).

[38] G. Juska, V. Dimastrodonato, L. O. Mereni, A. Gocalinska, and E. Pelucchi, Nat. Photonics 7, 527 (2013).

[39] S. Bose and V. Vedral, Phys. Rev. A 61, 040101 (2000).

[40] W. K. Wootters, Phys. Rev. Lett. 80, 2245 (1998).

[41] Cross dephasing in a QD is equivalent to dichroic scatterers for PDC sources, as discussed in Ref. [18] .

[42] D. F. V. James, P. G. Kwiat, W. J. Munro, and A. G. White, Phys. Rev. A 64, 052312 (2001).

[43] For a Werner-like state $\rho_{w}=p_{b}\left|\Phi^{+}\right\rangle\left\langle\Phi^{+}\right|+\left(1-p_{b}\right) I \otimes I / 4$, we have $f=\left\langle\Phi^{+}\left|\rho_{w}\right| \Phi^{+}\right\rangle=\left(1+3 p_{b}\right) / 4$, where $p_{b}$ is a weight of $\left|\Phi^{+}\right\rangle\left\langle\Phi^{+}\right|$in $\rho_{w}$. The Werner state violates the $\mathrm{CHSH}$ inequality for $p_{b}>1 / \sqrt{2}$, which corresponds to $f>(2+$ $3 \sqrt{2}) / 8 \sim 0.78$.

[44] E. M. Purcell, Phys. Rev. 69, 681(B.10) (1946). 